\title{
Modulation of fatty acid metabolism is involved in the alleviation of isoproterenol-induced rat heart failure by fenofibrate
}

\author{
PING LI ${ }^{1 *}$, SHIKE LUO $^{2 *}$, CHUNJI PAN ${ }^{1}$ and XIAOSHU CHENG ${ }^{1}$ \\ ${ }^{1}$ Department of Cardiovascular Medicine, The 2nd Affiliated Hospital of Nanchang University, Nanchang, Jiangxi 330006; \\ ${ }^{2}$ Department of Cardiology, The First Affiliated Hospital of Gannan Medical University, \\ Ganzhou, Jiangxi 341000, P.R. China
}

Received December 2, 2014; Accepted August 19, 2015

DOI: $10.3892 / \mathrm{mmr} .2015 .4466$

\begin{abstract}
Heart failure is a disease predominantly caused by an energy metabolic disorder in cardiomyocytes. The present study investigated the inhibitory effects of fenofibrate (FF) on isoproterenol (ISO)-induced hear failure in rats, and examined the underlying mechanisms. The rats were divided into $\mathrm{CON}$, ISO (HF model), FF and FF+ISO (HF animals pretreated with FF) groups. The cardiac structure and function of the rats were assessed, and contents of free fatty acids and glucose metabolic products were determined. In addition, myocardial cells were isolated from neonatal rats and used in vitro to investigate the mechanisms by which FF relieves heart failure. Western blot analysis was performed to quantify the expression levels of peroxisome proliferator-activated receptor (PPAR) $\alpha$ and uncoupling protein 2 (UCP2). FF effectively alleviated the ISO-induced cardiac structural damage, functional decline, and fatty acid and carbohydrate metabolic abnormalities. Compared with the ISO group, the serum levels of brain natriuretic peptide (BNP), free fatty acids, lactic acid and pyruvic acid were decreased in the FF animals. In the cultured myocardial cells, lactic acid and pyruvic acid contents were lower in the supernatants obtained from the FF animals, with lower levels of mitochondrial ROS production and cell necrosis, compared with the ISO group, whereas PPAR $\alpha$ upregulation and UCP2 downregulation occurred in the FF+ISO group. The results demonstrated that FF efficiently alleviated heart failure in the ISO-induced rat model, possibly via promoting fatty acid oxidation.
\end{abstract}

Correspondence to: Dr Ping Li, Department of Cardiovascular Medicine, The 2nd Affiliated Hospital of Nanchang University, 1 Min De Road, Nanchang, Jiangxi 330006, P.R. China

E-mail: lipingsydney@163.com

*Contributed equally

Key words: heart failure, fenofibrate, isoproterenol, peroxisome proliferator-activated receptor $\alpha$, uncoupling protein 2

\section{Introduction}

With the aging population and improvement in treatment for coronary heart disease, the prevalence and incidence of chronic heart failure (CHF) has increase steadily (1), particularly in developing countries. Heart failure is reported to have an annual mortality rate of $\sim 10 \%$ and a five-year mortality rate of $\sim 50 \%$, which results in a heavy economic burden on families and society $(2,3)$.

It is currently considered that myocardial energy metabolic disorder may be one of the most important factors preventing the success of heart failure treatment (4). The heart is arguably the most energy consuming organ in the body and it has been demonstrated that heart failure induces a reduction in energy production, promotes ventricular remodeling and impairs mitochondrial function, which further reduces energy production, leading to worsening heart function and subsequently aggravating the heart failure $(5,6)$.

In normal conditions, myocardial energy metabolism includes three processes: Substrate utilization, oxidative phosphorylation and adenosine triphosphate (ATP) transfer and utilization, and changes in either process may affect myocardial energy metabolism (7). When heart failure occurs, glucose is the preferred substrate to free fatty acids, resulting in an overall reduction of oxidative metabolism. If changes in energy substrate utilization are inhibited at the early stage of heart failure, ATP production may increase and the progression of heart failure hindered (8). It is well-known that peroxisome proliferator-activated receptors (PPARs) upregulate the expression of $\beta$ oxidase in myocardial cells, promote free fatty acid oxidation and increase ATP production (9). Therefore, activated PPAR inhibits cardiac hypertrophy, and PPAR activators are able to enhance oxidation and utilization of free fatty acids, preventing ventricular remodeling-induced cardiomyopathy or heart failure (10).

PPAR is a ligand-activated nuclear transcription factor, which belongs to the nuclear receptor superfamily, of which three subtypes have been described: PPAR $\alpha, \operatorname{PPAR} \beta$ and PPAR $\gamma$. PPAR $\alpha$ can be activated by synthetic ligands, including fibrates, and is predominantly associated with lipid metabolism, affecting target genes involved in fatty acid $\omega$-and $\beta$-oxidation (11). Fenofibrate (FF), a clofibric acid derivative lipid regulating drug, is an efficient PPAR $\alpha$ ligand with a long 
history of clinical use, reducing levels of low density lipoprotein, cholesterol and triglycerides, and increasing quantities of high-density lipoprotein (12).

During the process of heart failure, the expression of uncoupling protein (UCP) in myocardial cells is upregulated (13). This results in uncoupling of oxidative phosphorylation and a decrease in ATP production, further promoting the progression of heart failure. UCP2 is known to transport protons into the matrix, preventing their use in ATP synthesis, an effect that promotes free energy consumption of the electrochemical proton gradient, oxidative phosphorylation uncoupling and a reduction in ATP production (14). In addition, these events can reduce calcium excess and mitochondrial membrane potential, thereby inhibiting the production of reactive oxygen species (ROS) (15).

Therefore, heart failure may be treated by improving myocardial energy metabolism. In our previous study, an isoproterenol (ISO)-induced heart failure rat model was established (16). The present study aimed to further investigate the effects of FF on heart failure and energy metabolism, examine the underlying mechanisms using primary cultured neonatal rat cardiomyocytes and analyze the associations between PPAR $\alpha$, UCP2, myocardial energy metabolism and heart failure. The data aimed to provide a solid basis for understanding the effect of this drug class in the treatment of heart failure.

\section{Materials and methods}

Rats. A total of 80 male Sprague-Dawley rats (weight, 200-250 g; 10 weeks old) were provided by the Laboratory Animal Center of the Medical College of Nanchang University (Jiangxi, China). The rats were housed at $2-25^{\circ} \mathrm{C}$ in $40-80 \%$ humidity. The lighting was maintained at $12 \mathrm{~h} \mathrm{light/dark.} \mathrm{Male}$ and female rats were raised separately. The study procedures were reviewed and approved by the Animal Ethics Committee of the 2nd Affiliated Hospital of Nanchang University (Nanchang, China). All animals were allowed free access to food and water.

Reagents. Horseradish peroxidase (HRP)-conjugated rabbit anti-rat PPAR $\alpha$ (cat. no. sc-130640) and goat anti-rat UCP2 (cat. no. sc-390189) primary antibodies were purchased from Santa Cruz Biotechnology, Inc. (Santa Cruz, CA, USA). HRP-conjugated goat anti-rabbit (cat. no. sc-45101) and rabbit anti-goat (cat. no. sc-358922) IgG secondary antibodies were provided by Beijing Zhongshan Golden Bridge Biotechnology Co., Ltd. (Beijing, China).

Establishment of the heart failure animal model. The healthy inbred male SD rats were randomly divided into four groups $(\mathrm{n}=10)$ : $\mathrm{CON}$ (control), ISO, FF and FF+ISO groups. The rats in the CON group were fed a regular diet. In the ISO group, the rats were subcutaneously injected with $2.5 \mathrm{mg} / \mathrm{kg}$ ISO (Shanghai Harvest Pharmaceutical Co., Ltd., Shanghai, China) for 4 weeks to induce heart failure (HF model group). In the FF group, the animals were treated with $100 \mathrm{mg} / \mathrm{kg} / \mathrm{d}$ FF (France Libofuni pharmaceutical company, Libofuni, France) for 4 weeks. The rats in FF+ISO group were pretreated with $100 \mathrm{mg} / \mathrm{kg} / \mathrm{d} \mathrm{FF}$ for 4 weeks prior to the induction of heart failure, as described for the ISO group. At 4 weeks post-ISO treatment, echocardiography and histopathology examinations were performed to evaluate the heart failure model and assess the effects of FF on heart failure. In addition, the heart weight index was calculated to evaluate heart enlargement. The serum B-type brain natriuretic peptide (BNP; Kaiji, Nanjing, China) content in the three groups was also determined.

Echocardiography assessment of cardiac function in rats. Cardiac function in rats was assessed by measuring end-diastolic and end-systolic diameters, ejection fraction and shortening fraction of the left ventricle. Prior to analysis, the rats were anesthetized by intraperitoneal injection of $0.3 \%$ sodium pentobarbital solution $(10 \mathrm{ml} / \mathrm{kg})$ purchased from Sigma-Aldrich (Beijing, China). Ultrasonic detection of cardiac function indicators in the rats was performed on a Siemens Acuson Sequoia 512 echocardiographic instrument (Siemens Healthcare, Shanghai, China) with a $13 \mathrm{MHz}$ frequency wire probe (Siemens Healthcare) located near the sternum of the rat.

Preparation of myocardial pathological sections. The rats were sacrificed via an intraperitoneal injection of $0.3 \%$ pentobarbital sodium $(10 \mathrm{ml} / \mathrm{kg})$ and fixed on the plate. The chest was opened and the heart was exposed in full. The heart was cut open at the root of aorta and washed in cold saline. The surrounding connective tissues and blood vessels were cut off. The hearts were fixed with $10 \%$ formaldehyde solution (Jiancheng Bioengineering Institute, Nanjing, China) and embedded in paraffin (Jiancheng Bioengineering Institute). Using conventional methods, the heart specimens were dehydrated and embedded in paraffin. From the specimens, five slices ( $4 \mu \mathrm{m}$ in thickness) were cut along the cross section every other $1 \mathrm{~mm}$ in the long axis of the left ventricle, submitted to hematoxylin and eosin staining (Jiancheng Bioengineering Institute), and analyzed using optical microscopy (BX51; Olympus Corporation, Tokyo, Japan).

Determination of heart weight index. The rats were weighed, as described above, and fastened. Following sacrifice via intraperitoneal injection of $0.3 \%$ pentobarbital sodium $(10 \mathrm{ml} / \mathrm{kg})$, the chest was cut open using curved and straight scissors (325px; RWD Life Science, Shenzhen, China). Following thoracotomy, the fully exposed hearts were cut along aortic roots, and washed with precooled normal saline solution to remove residual blood. Subsequently, the connective tissues and blood vessels around the hearts were removed, and water was filtered out of the organs. The extracted hearts were weighed and the heart weight index was calculated for each, as a ratio of heart to body weight.

Determination of serum BNP. Rat serum BNP was determined using an enzyme-linked immunosorbent assay (ELISA) with a B-type BNP kit (Uscnk Life Science, Inc., Wuhan, China), according to the manufacturer's instructions. A 96-well ELISA plate was prepared by sequential incubation with $50 \mu 1$ anti-BNP antibodies, $100 \mu 1$ sample or standard and $50 \mu \mathrm{l}$ tracer solution. The mixture was dried and the plate was rinsed with phosphate-buffered saline (PBS) five times, followed by sequential incubation with $100 \mathrm{ml}$ prepared HRP-streptavidin solution (Santa Cruz Biotechnology, Inc.), 
$100 \mu 1$ TMB One-Step Substrate reagent (Fengshou Technology, Shanghai, China) and $100 \mu \mathrm{l}$ stop solution. Absorbance was read for samples and standards at $450 \mathrm{~nm}$ using an enzyme labelling instrument (Synergy HT; BioTek Instruments, Inc., Winooski, VT, USA). A standard curve (S-type) was plotted with known concentrations on the $\mathrm{x}$-axis and measured optical density values on the $y$-axis, on a semi-logarithmic scale. The quantities of BNP in samples were derived from the standard curve.

Determination of free fatty acid content in myocardial tissue and serum. Free fatty acid content was determined using solid-phase sandwich ELISA with a Free Fatty Acids kit (Nanjing Jiancheng Bioengineering Institute, Nanjing, China), according to the manufacturer's instructions. The samples or standard $(50 \mu \mathrm{l})$ were added to 96 -well plates for $1 \mathrm{~h}$, followed by incubation with $50 \mu \mathrm{l}$ biotin-labeled antibodies at $37^{\circ} \mathrm{C}$ for $1 \mathrm{~h}$. Following washing in PBS, the cells were incubated with $80 \mu \mathrm{l} \mathrm{HRP}$-streptavidin for $30 \mathrm{~min}$ at $37^{\circ} \mathrm{C}$. Subsequently, $50 \mu \mathrm{l}$ substrate $\mathrm{A}$ and $50 \mu \mathrm{l}$ substrate $\mathrm{B}$ (mixed gently by agitation) were added for detection at $37^{\circ} \mathrm{C}$ in the dark for $10 \mathrm{~min}$. Finally, $50 \mu 1$ stop solution was added and the absorbance was measured at $450 \mathrm{~nm}$, as described above. A standard curve was plotted to allow quantification of free fatty acids.

Determination of lactic acid and pyruvic acid contents. The lactic acid and pyruvic acid contents were determined using a Lactic Acid and Pyruvic Acid kit (Nanjing Jiancheng Bioengineering Institute), according to the manufacturer's instructions. Lactic acid and pyruvic acid were measured at 530 and $505 \mathrm{~nm}$, respectively, and the contents of lactic acid and pyruvic acid in the samples were calculated as follows:

Lactic acid content $=$ (absorbance of sample - absorbance of blank) / (absorbance of standard - absorbance of blank) x standard concentration $(3 \mu \mathrm{mol} / \mathrm{ml})$.

Pyruvic acid content $=$ (absorbance of sample - absorbance of blank) / (absorbance of standard - absorbance of blank) x standard concentration $(0.2 \mu \mathrm{mol} / \mathrm{ml})$.

Isolation, identification and in vitro culture of myocardial cells. Myocardial tissue was collected from healthy male Sprague-Dawley rats (1-3 days old), provided by the Laboratory Animal Center of Jiangxi University of Traditional Chinese Medicine (Jiangxi, China; 2010-0388). The rats were housed separately at $20-25^{\circ} \mathrm{C}$ in $60-70 \%$ humidity, and were sacrificed using intraperitoneal injection of $0.3 \%$ pentobarbital sodium $(10 \mathrm{ml} / \mathrm{kg})$. The chest was cut open using curved and straight scissors (325px; RWD Life Science). The tissues were digested with trypsin and collagenase, filtered and resuspended in Dulbecco's modified Eagle's medium (DMEM). According to the instructions contained in the Anti- $\alpha$ Skeletal Muscle Actin immunohistochemistry kit (Wuhan Boster Biological Technology, Ltd., Wuhan, China), the cells were labeled with anti- $\alpha$ skeletal muscle actin immunofluorescence at confluency, in a state of synchronizing pulse, and were used for myocardial beat detection under an inverted microscope.

Following isolation and purification, the myocardial cells $\left(1 \times 10^{6} / \mathrm{ml}\right)$ were incubated for $24 \mathrm{~h}$, and randomly divided into CON, ISO, FF and FF+ISO groups. In the ISO group, the cells were incubated for $24 \mathrm{~h}$ with $100 \mu \mathrm{mol} / 1 \mathrm{ISO}$. In the FF group, the cells were treated with $10 \mu \mathrm{mol} / 1 \mathrm{FF}$ for $25 \mathrm{~h}$. In the $\mathrm{FF}+\mathrm{ISO}$ group, the cells were pretreated with $10 \mu \mathrm{mol} / \mathrm{l} \mathrm{FF}$ for $1 \mathrm{~h}$ and cultured in the presence of $100 \mu \mathrm{mol} / 1 \mathrm{ISO}$ for $24 \mathrm{~h}$.

Fluorescence microscopy detection of mitochondrial ROS generation in the in vitro cell culture. Intracellular ROS oxidize non-fluorescent dichlorofluorescein (DCFH) into fluorescent DCF. Therefore, levels of intracellular ROS can be determined by assessing DCF fluorescence. In the present study, myocardial cells $\left(1 \times 10^{6} / \mathrm{ml}\right)$ were seeded into wells of a 96 -well plate $(15,000$ cells per well). Following innoculation, the cells were washed twice with Eagle's solution containing sugar, and were cultured with $25 \mu \mathrm{M}$ DCFH-DA (Jiancheng Bioengineering Institute) at $37^{\circ} \mathrm{C}$ for 30 mins. Following washes with Eagle's solution with sugar, the ISO and ISO-FF were administered. Fluorescence microscopy (excitation, 488 nm; emission, 525 nm; CHC, Olympus Corporation) was utilized to assess intracellular ROS levels.

Flow cytometric evaluation of cell apoptosis and necrosis in vitro. Using an Annexin V-Fluorescein isothiocyanate (FITC) Apoptosis Detection kit (Nanjing KGI Bio-tech. Co., Ltd., Nanjing, China), according to the manufacturer's instructions, the myocardial cells were digested with ethylene diamine tetraacetic acid-free trypsin and washed twice with PBS. Subsequently, $1-5 \times 10^{5}$ cells were successively incubated with $500 \mu \mathrm{l}$ binding buffer, $5 \mu \mathrm{l}$ Annexin V-FITC and $5 \mu \mathrm{l}$ propidium iodide, in the dark at room temperature for 5-15 min. Flow cytometry was then performed on a FACSCalibur (BD Biosciences, San Jose, CA, USA).

Western blot analysis of the expression levels of PPAR $\alpha$ and $U C P 2$. Once the culture medium was aspirated, 5-10x $10^{6}$ cells were collected with trypsin (Sigma-Aldrich) and washed with PBS. Lysis buffer (0.5 ml; Sigma-Aldrich), containing $50 \mathrm{mmol} / \mathrm{l}$ Tris $\mathrm{HCl}$ (pH 7.4), $150 \mathrm{mmol} / \mathrm{l} \mathrm{NaCl}, 1 \%$ Triton X-100, $1 \mathrm{mmol} / 1 \mathrm{Na}_{3} \mathrm{VO}_{4}$ and $1 \mathrm{mmol} / 1 \mathrm{NaF}$, was added to the cells, and the protein contents were determined using a Bicinchoninic Acid Protein Assay kit (Thermo Fisher Scientific, Inc.). Equal quantities of protein $(2 \mu \mathrm{g})$ were separated by $5 \%$ SDS-PAGE (Gefan Biotechnology, Shanghai, China) and transferred onto polyvinylidene fluoride membranes (Sigma-Aldrich) by electroblotting. The membranes were immersed in blocking buffer [5 g skimmed milk powder with $100 \mathrm{ml}$ Tris-buffered saline with $20 \%$ Tween (TBST)] and shaken for $1 \mathrm{~h}$ at room temperature or remained static at $4^{\circ} \mathrm{C}$ overnight. The membranes were then incubated with monoclonal rabbit anti-rat anti-PPAR $\alpha$ (1:200; Santa Cruz Biotechnology, Inc.; cat. no. sc-130640) and monoclonal goat anti-rat UCP2 (1:500) primary antibodies (Santa Cruz Biotechnology, Inc.; cat. no. sc-390189) for $2 \mathrm{~h}$ at room temperature. The membranes were washed with TBST (Nanjing Jiancheng Bioengineering Institute) for $10 \mathrm{mins}$ three times, followed by incubation with anti-rabbit (Santa Cruz Biotechnology, Inc.; cat. no. sc-45101) and anti-goat IgG (Santa Cruz Biotechnology, Inc.; cat. no. sc-358922) secondary antibodies conjugated to HRP $(1: 1,000)$ for $2 \mathrm{~h}$ at room temperature. The membranes were then washed with TBST for $10 \mathrm{mins}$ three times and the target bands were analyzed using a gel imaging system (Thermo Fisher Scientific, Inc., Waltham, MA, USA). 
Table I. Echocardiographic data of rats following treatment. Indicators of cardiac function of rats in the CON, FF, ISO and $\mathrm{FF}+\mathrm{ISO}$ groups were detected by echocardiography.

\begin{tabular}{llllll}
\hline Group & $\mathrm{n}$ & $\mathrm{DD}(\mathrm{mm})$ & $\mathrm{SD}(\mathrm{mm})$ & $\mathrm{EF}(\%)$ & FS $(\%)$ \\
\hline CON & 10 & $4.64 \pm 0.38$ & $2.68 \pm 0.30$ & $81.40 \pm 4.98$ & $47.00 \pm 5.00$ \\
FF & 10 & $4.69 \pm 0.33$ & $2.71 \pm 0.29$ & $79.84 \pm 5.76$ & $47.43 \pm 4.26$ \\
ISO & 10 & $6.46 \pm 0.58^{\mathrm{a}}$ & $4.74 \pm 0.29^{\mathrm{a}}$ & $54.20 \pm 5.40^{\mathrm{a}}$ & $28.60 \pm 3.05^{\mathrm{a}}$ \\
FF+ISO & 10 & $5.44 \pm 0.36^{\mathrm{b}, \mathrm{d}}$ & $3.66 \pm 0.43^{\mathrm{a}, \mathrm{c}}$ & $71.60 \pm 2.70^{\mathrm{a}, \mathrm{c}}$ & $35.20 \pm 3.49^{\mathrm{a}, \mathrm{d}}$ \\
\hline
\end{tabular}

Data are expressed as the mean \pm standard deviation. ${ }^{\mathrm{a}} \mathrm{P}<0.01$ and ${ }^{\mathrm{b}} \mathrm{P}<0.05$, compared with the CON group; ${ }^{\mathrm{c}} \mathrm{P}<0.01$ and ${ }^{\mathrm{d}} \mathrm{P}<0.05$, compared with the ISO group. CON, control; FF, fenofibrate; ISO, isoproterenol; DD, left ventricular diastolic diameter; SD, left ventricular systolic diameter; EF, left ventricular ejection fraction; FS, left ventricular fractional shortening.

Statistical analysis. Data are expressed as the mean \pm standard deviation. Data analysis was performed using the Statistical Package for the Social Sciences (SPSS) 18.0 software (SPSS, Inc., Chicago, IL, USA). One way analysis of variance (was used for intergroup comparisons and $\mathrm{P}<0.05$ was considered to indicate a statistically significant difference.

\section{Results}

FF effectively alleviates ISO-induced heart failure in rats. Daily subcutaneous injections of ISO for 4 weeks significantly reduced myocardial systolic function and enlarged ventricles in the rats. In addition, ISO treatment resulted in cardiomyocyte hypertrophy, with necrosis and hyperplasia of myocardial interstitial connective tissue observed. Following 4 weeks of ISO administration, the rats exhibited loss of appetite, had dry and yellowish fur and exhibited manifestations of heart failure, including slow weight increase, breathing difficulties and reduced activity.

Echocardiography data revealed that, compared with the control animals, left ventricular diameter and systolic function in the ISO group were significantly enlarged and decreased, respectively (Table I). End-diastolic and end-systolic diameters, ejection fraction and shortening fraction of the left ventricle were all significantly improved in the FF+ISO group, compared with the ISO group. No statistical significant differences were observed in the values obtained for the FF group, compared with the control animals (Table I).

Gross examination revealed a dark purple heart surface in the rats of the ISO group, whereas the hearts of the control animals was ruddy. Following analysis under microscopy, cardiac sections from the ISO group exhibited overt hypertrophy, acidophilic degeneration or necrosis of a number of myocardial cells. In addition, focal or diffuse lesions, predominantly distributed in the left ventricular wall, were observed in these animals, with certain rats exhibiting cord-like hyperplasia of myocardial interstitial connective tissue. The pathological damage observed in the myocardial tissues was significantly reduced in the $\mathrm{FF}+\mathrm{ISO}$ group, compared with animals treated with ISO only. As expected, the myocardial cells were of normal size and neatly arranged in the control group (Fig. 1). These data demonstrated that FF reversed the ISO-induced myocardial damage in rats.
Table II. HWI and serum levels of BNP in rats following treatment.

\begin{tabular}{llll}
\hline Group & $\mathrm{n}$ & HWI $(\mathrm{mg} / \mathrm{g})$ & $\mathrm{BNP}(\mathrm{ng} / \mathrm{ml})$ \\
\hline CON & 10 & $2.13 \pm 0.15$ & $0.39 \pm 0.03$ \\
FF & 10 & $2.11 \pm 0.13$ & $0.40 \pm 0.05$ \\
ISO & 10 & $3.58 \pm 0.08^{\mathrm{a}}$ & $4.86 \pm 0.12^{\mathrm{a}}$ \\
FF+ISO & 10 & $3.16 \pm 0.12^{\mathrm{b}, \mathrm{c}}$ & $1.79 \pm 0.09^{\mathrm{a}, \mathrm{c}}$ \\
\hline
\end{tabular}

Data are expressed as the mean \pm standard deviation. ${ }^{\mathrm{a}} \mathrm{P}<0.01$ and ${ }^{\mathrm{b}} \mathrm{P}<0.05$, compared with the $\mathrm{CON}$ group; ${ }^{\mathrm{C}} \mathrm{P}<0.01$, compared with the ISO group. CON, control; FF, fenofibrate; ISO, isoproterenol; HWI, heart weight index; BMP, B-type brain natriuretic peptide.

Table III. FFA content in the serum and myocardial tissues of the rats.

\begin{tabular}{lccc}
\hline Group & $\mathrm{n}$ & $\begin{array}{c}\text { FFA in serum } \\
(\mu \mathrm{mol} / \mathrm{l})\end{array}$ & $\begin{array}{c}\text { FFA in myocardial } \\
\text { tissue }(\mu \mathrm{mol} / \mathrm{gprot})\end{array}$ \\
\hline CON & 10 & $480.32 \pm 44.71$ & $493.74 \pm 17.72$ \\
FF & 10 & $483.97 \pm 45.81$ & $497.64 \pm 20.55$ \\
ISO & 10 & $782.76 \pm 65.98^{\mathrm{a}}$ & $650.42 \pm 54.38^{\mathrm{a}}$ \\
FF+ISO & 10 & $596.62 \pm 112.18^{\mathrm{b}, \mathrm{c}}$ & $565.70 \pm 57.55^{\mathrm{b}, \mathrm{d}}$ \\
\hline
\end{tabular}

Data are expressed as the mean \pm standard deviation. ${ }^{a} \mathrm{P}<0.01$ and ${ }^{\mathrm{b}} \mathrm{P}<0.05$, compared with the CON group, ${ }^{\mathrm{c}} \mathrm{P}<0.01$ and ${ }^{\mathrm{d}} \mathrm{P}<0.05$, compared with the ISO group. CON, control; FF, fenofibrate; ISO, isoproterenol; FFA, free fatty acid.

The heart weight index, defined as the ratio of heart to body weight, was higher in the ISO and FF+ISO groups than in the control group. However, the heart weight index was lower in FF+ISO group than the ISO group, indicating a relief of the ISO-induced heart enlargement by FF. A similar trend was observed in the serum levels of BNP, which were significantly higher in the ISO and FF+ISO groups, compared with the control, but significantly reduced in the FF+ISO group, compared with the ISO group (Table II). Of note, similar values were obtained in the CON and FF groups for heart weight index and serum levels of B-type BNP (Table II). 
Table IV. Lactate and pyruvic acid contents in myocardial tissue of rats and in supernatants collected from in vitro cultured cardiac cells.

\begin{tabular}{|c|c|c|c|c|c|}
\hline \multirow[b]{2}{*}{ Group } & \multirow[b]{2}{*}{$\mathrm{n}$} & \multicolumn{2}{|c|}{ In vivo myocardial tissue } & \multicolumn{2}{|c|}{ In vivo cultured cardiac cells } \\
\hline & & $\begin{array}{c}\text { Lactic acid } \\
\text { (mmol/gprot) }\end{array}$ & $\begin{array}{l}\text { Pyruvic acid } \\
\text { ( } \mu \mathrm{mol} / \mathrm{gprot})\end{array}$ & $\begin{array}{l}\text { Lactic acid } \\
\qquad(\mu \mathrm{mol} / 1)\end{array}$ & $\begin{array}{l}\text { Pyruvic acid } \\
\quad(\mathrm{nmol} / \mathrm{l})\end{array}$ \\
\hline $\mathrm{CON}$ & 10 & $0.22 \pm 0.34$ & $0.26 \pm 0.01$ & $4.90 \pm 0.05$ & $212.25 \pm 0.86$ \\
\hline $\mathrm{FF}$ & 10 & $0.43 \pm 0.25$ & $0.27 \pm 0.03$ & $4.89 \pm 0.14$ & $216.40 \pm 6.11$ \\
\hline ISO & 10 & $0.66 \pm 0.65^{\mathrm{a}}$ & $1.17 \pm 0.09^{\mathrm{a}}$ & $10.57 \pm 1.66^{\mathrm{a}}$ & $549.63 \pm 2.41^{\mathrm{a}}$ \\
\hline $\mathrm{FF}+\mathrm{ISO}$ & 10 & $0.37 \pm 0.52^{\mathrm{a}, \mathrm{c}}$ & $0.62 \pm 0.01^{\mathrm{a}, \mathrm{b}}$ & $6.88 \pm 0.56^{\mathrm{a}, \mathrm{b}}$ & $335.49 \pm 1.82^{\mathrm{a}, \mathrm{b}}$ \\
\hline
\end{tabular}

Data are expressed as the mean \pm standard deviation. ${ }^{\mathrm{a}} \mathrm{P}<0.05$, compared with the $\mathrm{CON}$ group; ${ }^{\mathrm{b}} \mathrm{P}<0.01$ and ${ }^{\mathrm{c}} \mathrm{P}<0.05$, compared with the ISO group. CON, control; FF, fenofibrate; ISO, isoproterenol.

A

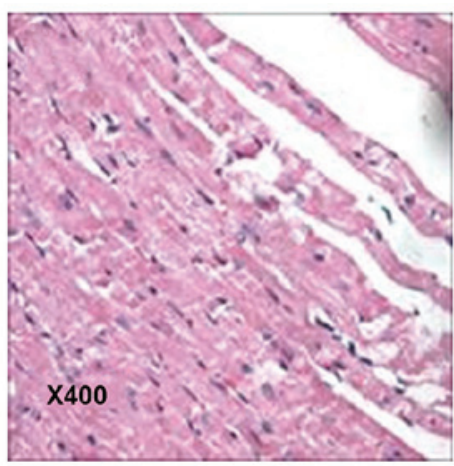

B

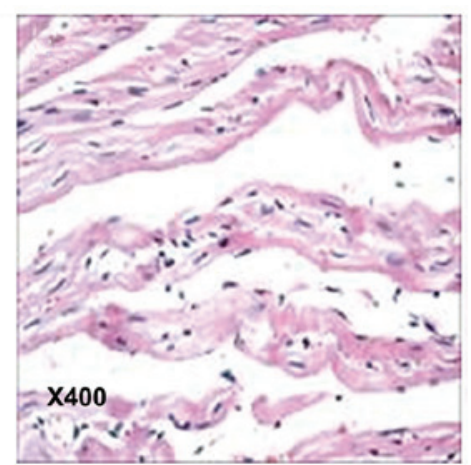

C

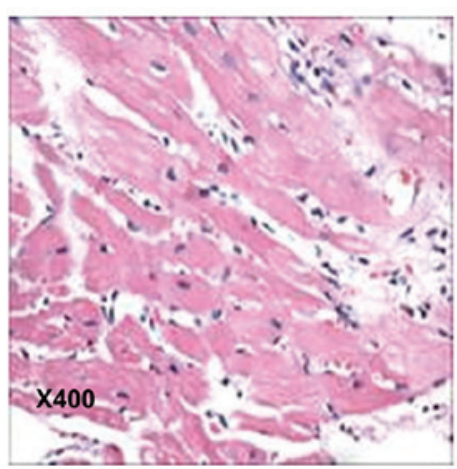

Figure 1. Myocardial biopsy of rat myocardial tissues stained with hematoxylin and eosin. (A) control group; (B) fenofibrate+ISO group; (C) ISO group. For all groups, $\mathrm{n}=10$. ISO, isoproterenol.
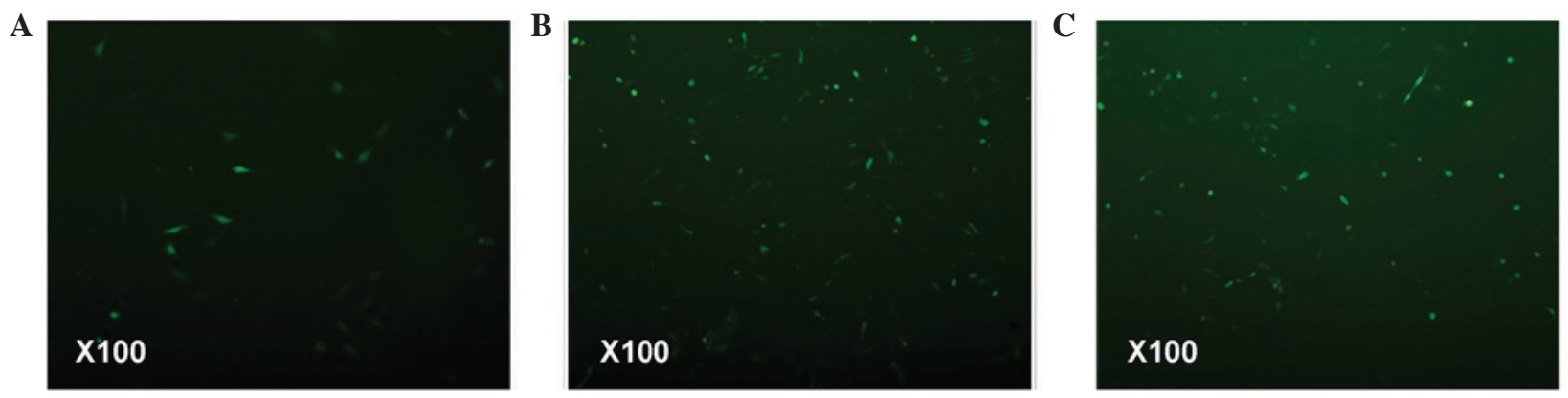

Figure 2. Reactive oxygen species generation, detected using fluorescence microscopy. (A) Control group, (B) fenofibrate+ISO group and (C) ISO group. ( $\mathrm{n}=10 /$ group). ISO, isoproterenol.

FF effectively alleviates ISO-induced metabolic abnormalities in a rat model of heart failure. Free fatty acid levels in the serum and myocardial tissue, and lactate acid and pyruvic acid contents in the myocardial tissue were determined to assess fatty acid and carbohydrate metabolism in rats following treatment. Free fatty acids reflect the efficiency of fatty acid metabolism, with high contents indicating low metabolic efficiency. In the present study, free fatty acid contents in the serum and myocardial tissue were higher in the ISO group, compared with the FF+ISO group, with control animals exhibiting the lowest values. No statistically significant differences were observed between the CON and FF groups (Table III). These findings were in agreement with the histological data, and provided evidence supporting fatty acid metabolic dysfunction in ISO-induced heart failure rats, as well as effective improvement in fatty acid metabolism following FF treatment.

The levels of lactic acid and pyruvic acid, glucose metabolism intermediates, were detected using spectrophotometry. The data revealed that lactic acid and pyruvic acid were higher in content in the myocardial tissues obtained from the ISO group, compared with those from the FF+ISO group, with the control group animals exhibiting the lowest levels of these metabolites (Table IV). These findings indicated an increase in anaerobic glycolysis in the ISO-induced heart failure rats, 
A

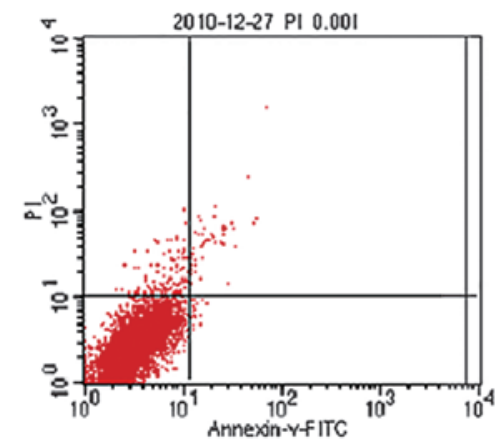

B

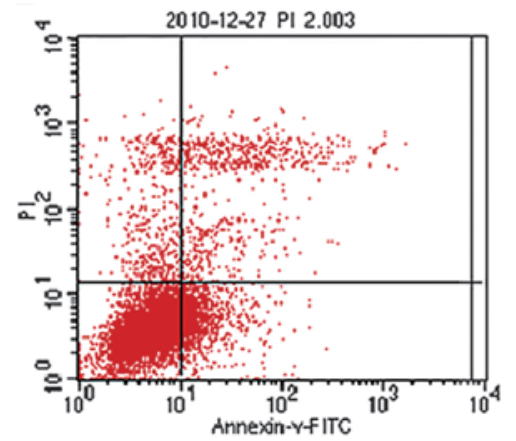

C

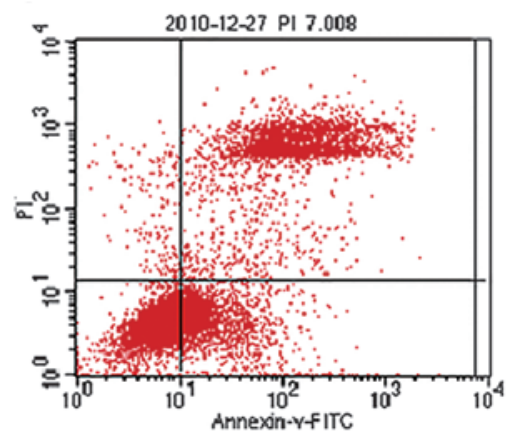

Figure 3. Cell apoptosis/necrosis detection using Annexin V/FITC flow cytometry. (A) Control group; (B) fenofibrate+ISO group and (C) ISO group.

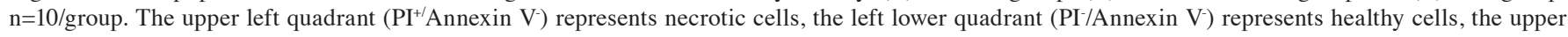

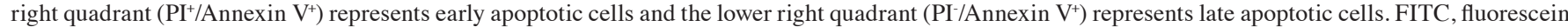
isothiocyanate; PI, propidium iodide; ISO, isoproterenol.
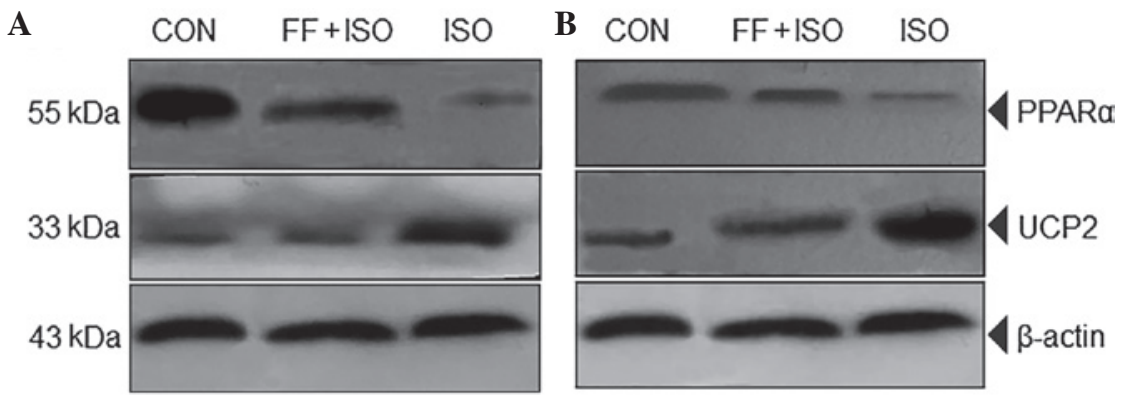

Figure 4. Expression of PPAR $\alpha$ and UCP2. (A) Neonatal rat cardiomyocytes; (B) rat myocardial tissue. Samples from the CON, FF+ISO and ISO groups were analyzed (n=10/group). CON, control; FF, fenofibrate; ISO, isoproterenol; PPAR, peroxisome proliferator-activated receptor; UCP2, uncoupling protein 2.

which was altered by FF treatment. As shown in Table IV, animals in FF and CON groups exhibited similar values for all the parameters investigated.

FF inhibits ISO-induced increases of anaerobic glycolysis, ROS levels and cell necrosis in vitro. In order to examine the mechanisms underlying the effects of FF on heart failure, the present study isolated, purified and identified neonatal rat myocardial cells for in vitro investigations. Significantly reduced lactic acid and pyruvic acid contents were found in the $\mathrm{FF}+\mathrm{ISO}$ group, compared with the ISO group $(\mathrm{P}<0.05)$, with the control animals exhibiting the lowest values (Table IV). The high lactic acid and pyruvic acid contents indicated that ISO induced abnormal glucose metabolism and anaerobic glycolysis in the in vitro myocardial cell culture, the effects of which were inhibited by treatment with FF. The cells treated with FF alone exhibited similar lactic acid and pyruvic acid contents as detected in the control cells (Table IV).

To investigate whether abnormal cellular energy metabolism results in oxidative phosphorylation in mitochondria and causes abnormal ATP production, the present study subsequently examined mitochondrial ROS. The accumulation of ROS indicates decreased oxidative phosphorylation, which promotes the enhancement of mitochondrial membrane potential. The fluorescence microscopy data revealed that the ISO-treated cardiomyocytes were more fluorescent, indicating high ROS levels in this group (Fig. 2). Notably, FF pretreatment decreased myocardial cell fluorescence. However, the fluorescence intensity observed in the myocardial cells of the $\mathrm{FF}+\mathrm{ISO}$ group remained higher, compared with hat of the control cells. These findings suggested that FF reversed the ISO-induced decrease of oxidative phosphorylation.

ROS accumulation and mitochondrial activation can cause cell apoptosis or necrosis. The flow cytometry data demonstrated that treatment with ISO resulted in marked cell death, and this effect was markedly reduced following FF pretreatment. These findings suggested that ISO-induced abnormal energy metabolism ultimately resulted in cell necrosis, and that FF treatment restored energy metabolism and reduced cell necrosis (Fig. 3).

ISO and FF regulate the expression levels of PPAR $\alpha$ and $U C P 2$. To understand the mechanisms by which FF alleviates ISO-induced cell energy metabolic disorder, necrosis and heart failure in rats, the present study assessed the expression levels of PPAR $\alpha$ and UCP2 using western blot analysis. The expression of PPAR $\alpha$ was significantly increased and that of UCP2 was significantly reduced in the myocardial cells of the FF+ISO group, compared with the ISO groups, in vitro (Fig. 4A) and in vivo (Fig. 4B). These data demonstrated that FF, a PPAR $\alpha$ ligand, may promote the oxidation of fatty acids by $\beta$-oxidase by PPAR $\alpha$ activation, therefore providing energy for myocardial cells. In addition, FF may downregulate the ISO-induced expression of UCP2, inhibit the uncoupling of oxidative phosphorylation caused by ISO and provide more ATP or energy in myocardial cells. 


\section{Discussion}

Heart failure is a major cause of disability and mortality, with a five-year mortality rate as high as $50 \%$ (17). Its predominant etiologic mechanism includes myocardial energy metabolic disorder (16). Even for a healthy heart, energy substrates can only provide $\sim 25 \%$ energy, far less than the body demands. Thus, a small variation in the efficiency of energy production and utilization can significantly affect myocardial energy levels (18).

In order to understand the occurrence of heart failure and the mechanisms underlying the therapeutic effects of FF in the treatment of heart failure, the present study established and investigated a heart failure rat model, in vivo and in vitro, for energy utilization. It is suggested that heart failure may be accompanied by excessive activation of the sympathetic nervous system and increased relative myocardial ischemia and hypoxia, which is possibly associated with catecholamine-induced lipid peroxidation, membrane permeability changes and intracellular calcium overload (19). Large doses of ISO in rats results in diffuse or focal myocardial necrosis, cardiac enlargement, calcium accumulation in myocardial tissue and enhancement of lipid peroxidation (20). In this study, daily subcutaneous $2.5 \mathrm{mg} / \mathrm{kg}$ doses of ISO were injected each day into rats for 4 weeks. At the end of treatment, the survival rate was high and the majority of the rats exhibited heart failure symptoms, including yellowish hair, appetite loss, slow weight increase, breathing accompanied by wheezing and reduced activity. Cardiac function, heart weight index and serum levels of BNP in rats were significantly altered, suggesting a successful rat model of heart failure.

During the occurrence and development of $\mathrm{CHF}$, substrates for myocardial energy metabolism change from fatty acids to glucose, resulting in increased consumption of ATP. However, the decrease in fatty acid oxidation cannot be fully compensated by the enhanced glucose oxidation. This results in insufficient energy production and further aggravates heart failure (8). Therefore, CHF mechanisms may include changes in mitochondrial energy source, depending on the expression of enzymes in the fatty acid oxidation pathway ( $\beta$-oxidation is reduced). It has been demonstrated that the reduction of fatty acid utilization in myocardial cells with heart failure may be associated with decreased expression of $\beta$-oxidase $(21,22)$. $\beta$-oxidase promotes $\beta$-oxidation of fatty acids in the mitochondria of myocardial cells, resulting in increased ATP, and activated PPAR $\alpha$ regulates myocardial fatty acid $\beta$-oxidase and promotes fatty acid uptake, activation and metabolism in myocardial cells (23). However, during heart failure, the expression of PPAR $\alpha$ is also decreased in the mitochondria of myocardial cells, (24). which may be a major reason why the myocardium changes its preferred energy source from fatty acid to glucose.

The present study revealed decreased expression levels of PPAR $\alpha$ in the ISO and FF+ISO groups, compared with the normal myocardial tissue. Of note, the ISO group exhibited the lowest expression of this enzyme. In contrast, fatty acid contents in the serum and myocardial tissue were highest in the ISO group and lowest in the control group, with the $\mathrm{FF}+\mathrm{ISO}$ group between. These data suggested that the decreased expression of PPAR $\alpha$ in myocardial tissue during heart failure in rats of the ISO group reduced PPAR $\alpha$ activity. Notably, the data revealed significantly improved cardiac function, as assessed by echocardiography, myocardial biopsy and serum BNP content in the FF+ISO group. These findings indicated that FF, as a highly selective PPAR $\alpha$ agonist, may increase fatty acid $\beta$-oxidation enzymes by activating PPAR $\alpha$, promoting fatty acid oxidation in the mitochondria, regulating myocardial energy metabolism, and improving ventricular remodeling.

Uncoupling proteins (UCPs) are constituents of the mitochondrial inner membrane, of which they can lower the electrochemical gradient by promoting $\mathrm{H}^{+}$permeability between the mitochondrial inner membrane and substrate, and therefore, decrease ATP production. The UCP family includes UCP1-5, UCP2 and UCP3, which are predominantly expressed in the cardiovascular system (25), and the expression of UCP2 has been observed to increase when heart failure occurs. Noma et al (26) reported increased expression of UCP2 and decreased ATP production with the progression of heart failure in a mouse model of aortic regurgitation, indicating that UCP2-induced ATP deficiency is a predominant cause of heart failure.

In addition, previous studies have demonstrated that the myocardial protein expression of UCP2 is positively correlated with serum free fatty acid content, and it is known that high plasma free fatty acid content can promote the myocardial expression of UCP2 (27).

The data of the present study demonstrated that serum free fatty acid content was reduced and myocardial UCP2 protein was downregulated in the FF+ISO group, compared with the ISO group, however, ventricular remodeling and cardiac function were significantly improved in the $\mathrm{FF}+\mathrm{ISO}$ group in comparison with the ISO group. Therefore, in agreement with our previous work $(28,29)$, this data indicated that the regulation of the expression of UCP2 may be involved in the mechanism by which lipid-lowering drugs relieve heart failure.

In the present study, only one dose of FF was examined in the in vivo and in vitro experiments, and achieved partial inhibition of the effects of ISO. The hemodynamic effects of FF are not clear, as data regarding the blood pressure and heart rates of the rats are limited. Future investigations are required to assess higher, but not toxic doses, also in other animal models.

Overall, the in vivo and in vitro results of the present study demonstrated that FF efficiently alleviated ISO-induced HF in rats, which was possibly due to the promotion of fatty acid oxidative metabolism. In addition, UCP2-induced uncoupling of oxidative phosphorylation may be involved in the FF effect.

\section{Acknowledgements}

This study was supported by grants from the National Natural Science Foundation of China (grant no, 1140019), the Natural Science Foundation of JiangXi Province (grant no. 20122BAB205007) and the Science and Technology Support Project of Nanchang (grant no. 49-10).

\section{References}

1. Stewart S, MacIntyre K, Capewell S and McMurray JJ: Heart failure and the aging population: An increasing burden in the 21st century?. Heart 89: 49-53, 2003 
2. Bettari L, Fiuzat M, Felker GM and O'Connor CM: Significance of hyponatremia in heart failure. Heart Fail Rev 17: 17-26, 2012.

3. Dayer M and Cowie MR: Heart failure: Diagnosis and healthcare burden. Clin Med 4: 13-18, 2004.

4. Sinatra ST: Metabolic cardiology: The missing link in cardiovascular disease. Altern Ther Health Med 15: 48-50, 2009.

5. Herrmann G and G D: The chemical nature of heart failure. Ann Intern Med 12: 1233-1244, 1939.

6. Neubauer S: The failing heart-an engine out of fuel. N Engl J Med 356: 1140-1151, 2007.

7. J I: ATP and the heart [M]. Kluwer academic publishers USA: pp197-pp216, 2002.

8. Nagoshi T, Yoshimura M, Rosano GMC, Lopaschuk GD and Mochizuki S: Optimization of cardiac metabolism in heart failure. Curr Pharm Des 17: 3846-3853, 2011.

9. Kim T and Yang Q: Peroxisome-proliferator-activated receptors regulate redox signaling in the cardiovascular system. World J Cardiol 5: 164-174, 2013.

10. Planavila A, Rodriguez-Calvo R, Jové M, Michalik L, Wahli W, Laguna JC and Vázquez-Carrera M: Peroxisome proliferator-activated receptor beta/delta activation inhibits hypertrophy in neonatal rat cardiomyocytes. Cardiovasc Res 65: 832-841, 2005

11. Umemoto T and Fujiki Y: Ligand-dependent nucleo-cytoplasmic shuttling of peroxisome proliferator-activated receptors, PPAR $\alpha$ and PPAR $\gamma$. Genes Cells 17: 576-596, 2012

12. Scott R, O'Brien R, Fulcher G, Pardy C, D'Emden M, Tse D, Taskinen MR, Ehnholm C and Keech A; Fenofibrate Intervention and Event Lowering in Diabetes (FIELD) Study Investigators: Effects of fenofibrate treatment on cardiovascular disease risk in 9,795 individuals with type 2 diabetes and various components of the metabolic syndrome: The fenofibrate intervention and event lowering in diabetes (FIELD) study. Diabetes Care 32: 493-498, 2009.

13. Teshima Y, Akao M, Jones SP and Marbán E: Uncoupling protein-2 overexpression inhibits mitochondrial death pathway in cardiomyocytes. Circ Res 93: 192-200, 2003.

14. Echtay KS: Mitochondrial uncoupling proteins - what is their physiological role? Free Radic Biol Med 43: 1351-1371, 2007.

15. Deng S, Yang Y, Han Y, Li X, Wang X, Zhang Z and Wang Y: UCP2 inhibits ROS-mediated apoptosis in A549 under hypoxic conditions. PLoS One 7: e30714, 2012.

16. Ingwall JS: Energy metabolism in heart failure and remodelling. Cardiovasc Res 81: 412-419, 2009.

17. Askoxylakis V, Thieke C, Pleger ST, Most P, Tanner J, Lindel K, Katus HA, Debus J and Bischof M: Long-term survival of cancer patients compared to heart failure and stroke: A systematic review. BMC Cancer 10: 105, 2010.
18. Joiner ML, Koval OM, Li J, He BJ, Allamargot C, Gao Z, Luczak ED, Hall DD, Fink BD, Chen B, et al: CaMKII determines mitochondrial stress responses in heart. Nature 491: 269-273, 2012.

19. Andalib S, Shayanfar A, Khorrami A, Maleki-Dijazi N and Garjani A: Atorvastatin reduces the myocardial content of coenzyme Q10 in isoproterenol-induced heart failure in rats. Drug Res (Stuttg) 64: 246-250, 2014

20. Heymes C, Bendall JK, Ratajczak P, Cave AC, Samuel JL, Hasenfuss G and Shah AM: Increased myocardial NADPH oxidase activity in human heart failure. J Am Coll Cardiol 41: 2164-2171, 2003

21. Burkart EM, Sambandam N, Han X, Gross RW, Courtois M, Gierasch CM, Shoghi K, Welch MJ and Kelly DP: Nuclear receptors PPARbeta/delta and PPARalpha direct distinct metabolic regulatory programs in the mouse heart. J Clin Invest 117: 3930-3939, 2007.

22. Wolins NE, Quaynor BK, Skinner JR, Tzekov A, Croce MA, Gropler MC, Varma V, Yao-Borengasser A, Rasouli N, Kern PA, et al: OXPAT/PAT-1 is a PPAR-induced lipid droplet protein that promotes fatty acid utilization. Diabetes 55: 3418-3428, 2006.

23. Sack MN, Rader TA, Park S, Bastin J, McCune SA and Kelly DP: Fatty acid oxidation enzyme gene expression is downregulated in the failing heart. Circulation 94: 2837-2842, 1996.

24. Garnier A, Fortin D, Deloménie C, Momken I, Veksler V and Ventura-Clapier R: Depressed mitochondrial transcription factors and oxidative capacity in rat failing cardiac and skeletal muscles. J Physiol 551: 491-501, 2003.

25. Murray AJ, Anderson RE, Watson GC, Radda GK and Clarke K: Uncoupling proteins in human heart. Lancet 364: 1786-1788, 2004.

26. Noma T, Nishiyama A, Mizushige K, Murakami K, Tsuji T, Kohno M, Rahman M, Fukui T, Abe Y and Kimura S: Possible role of uncoupling protein in regulation of myocardial energy metabolism in aortic regurgitation model rats. FASEB J 15: 1206-1208, 2001.

27. Young ME, Patil S, Ying J, Depre C, Ahuja HS, Shipley GL, Stepkowski SM, Davies PJ and Taegtmeyer H: Uncoupling protein 3 transcription is regulated by peroxisome proliferator-activated receptor (alpha) in the adult rodent heart. FASEB J 15: 833-845, 2001.

28. Li P, Yin K, Luo SK and Cheng XS: Effect of uncoupling protein-2 on angiotensin II induced reactive oxygen species generation in mitochondria. Chinese Journal of Hypertension 18: 940-945, 2010 (In Chinese)

29. Luo SK, Li P and Cheng XS: Establishment of model of isoprenaline-induced chronic heart failure in rats. Chongqing Yixue 41: 352-354, 2012 